\title{
A METHOD TO COMPUTE SEGRE CLASSES OF SUBSCHEMES OF PROJECTIVE SPACE
}

\author{
DAVID EKLUND, CHRISTINE JOST, AND CHRIS PETERSON
}

\begin{abstract}
We present a method to compute the degrees of the Segre classes of a subscheme of complex projective space. The method is based on generic residuation and intersection theory. We provide a symbolic implementation using the software system Macaulay2 and a numerical implementation using the software package Bertini.
\end{abstract}

\section{INTRODUCTION}

Segre classes are generalizations of characteristic classes of vector bundles and they occur frequently in intersection theory. Many problems in enumerative geometry may be solved by computing the Segre classes of an algebraic scheme. Given an $n$-dimensional subscheme $Z$ of complex projective space there are $n+1$ Segre classes of $Z$. The $i^{t h}$ Segre class is a rational equivalence class of codimension $i$ cycles on $Z$. Thus a Segre class may be represented as a weighted sum of irreducible subvarieties $V_{1}, \ldots, V_{m}$ of $Z$. The degree of a Segre class is the corresponding weighted sum of the degrees of the projective varieties $V_{1}, \ldots, V_{m}$.

In this paper we present a method to compute the degrees of the Segre classes of $Z$, given an ideal defining $Z$. The procedure is based on the intersection theory of Fulton and MacPherson. More specifically, we prove a Bézout like theorem that involves the Segre classes of $Z$ and a residual scheme to $Z$. The degree of the residual may be computed providing intersection-theoretic information on the Segre classes. This enables us to compute the degrees of these classes.

If $Z$ is smooth, the degrees of the Segre classes of $Z$ carry the same information as the degrees of the Chern classes of the tangent bundle of $Z$. For instance, when $Z$ is smooth, the degree of the top Chern class, which is equal to the topological Euler characteristic of $Z$, can be computed from the degrees of the Segre classes of $Z$. Hence one can compute the topological Euler characteristic using our procedure (provided that the input ideal defines a smooth scheme). The relationship between the degrees of the Segre classes of $Z$ and the Chern classes of the tangent bundle of $Z$ may be extended to the non-smooth case via the so-called Chern-Fulton classes of $Z$. In case $Z$ is smooth, the Chern-Fulton classes coincide with the Chern classes of the tangent bundle.

We would like to mention two interesting features of our method. One feature is that the algorithm is completely elementary in that it requires no knowledge of intersection theory to understand the steps in the procedure and only very basic

2000 Mathematics Subject Classification. 13Pxx, 14Qxx, 14C17, 65H10, $65 \mathrm{E} 05$.

Key words and phrases. Segre classes, computational algebraic geometry, numerical homotopy methods. 
background in algebraic geometry. The method therefore provides a way of understanding the computation of Segre classes from an elementary point of view. In addition, the algorithm is easy to implement. Another feature is the fact that our method is implementable in a numerical setting via numerical homotopy methods, see 11] for an overview of this area. This allows the method to be applied in settings that can be time consuming (and even out of reach) of current symbolic methods. One such setting is when the generating set, for an ideal determining $Z$, has complicated coefficients. An additional setting where current numerical methods can sometimes obtain useful information about the degrees of Segre classes, beyond the reach of current symbolic methods, is when $Z$ is a reduced scheme of high codimension. These gains come through numerical approximation and parallelization (but at the expense of exactness).

The procedure presented in this paper has been implemented in the symbolic setting using the software system Macaulay2 [7] and in the numerical setting using the software package Bertini [3]. Both implementations are available at http://www.math.su.se/ jost/segreimplementation.htm. Initial experiments, involving subvarieties of relatively large dimension and codimension, show a great deal of promise for the algorithm in the numerical setting.

In the paper [1] Aluffi formulates an algorithm that also computes the degrees of the Segre classes of a subscheme $Z$ of projective space. In addition he shows how to relate the computation of the so-called Chern-Schwartz-MacPherson classes of a subscheme of projective space to the computation of the degrees of certain Segre classes. In the present paper we present an alternative method to Aluffi's. Though the two are closely related, they have a rather different computational behavior and seem to complement each other well (see Section 6 for some examples). Apart from the difference in computing speed in various cases one may ask what is the need for another method with the same output as an existing method. One answer is that our approach is different and therefore sheds new light on the problem of computing Segre classes. But more importantly we would answer by repeating the two features mentioned above, namely that our method is elementary and that it is readily amenable to numerical computation.

The paper is organized as follows. In Section 2 we give the basic definitions and state a theorem from intersection theory. In Section 3 we derive a recursive formula for Segre classes which is the basis of our method. The procedure to compute Segre classes is presented in Section 4. Some examples are given in Section [5 and in Section [6 we give a list of run times on examples comparing our method to other algorithms.

The results of this paper are generalizations and variants of the results in 2, 4] to the setting of subschemes of projective space.

\section{ACKNOWLEDGMENTS}

We would like to thank Paolo Aluffi and Sandra Di Rocco for their useful comments and encouragement. We thank Jon Hauenstein for pointing out how we could utilize efficient numerical methods and also for his help with running examples in Bertini. Finally, we thank the Institut Mittag-Leffler for their wonderful research environment that facilitated the completion of this paper. 


\section{BaCkground In InTERSECtion THEORY}

We start by going through some concepts and results from intersection theory. For this paper, the main reference on matters of intersection theory is Fulton's book [5].

2.1. Notation. Let $Y$ be an algebraic scheme over $\mathbb{C}$ of dimension $n$. By a subscheme of $Y$ we will mean a closed subscheme. We will denote by $C_{p}(Y)$ the free Abelian group on irreducible $p$-dimensional subvarieties of $Y$. The $p^{\text {th }}$ Chow group of $Y$ is the quotient of $C_{p}(Y)$ by the cycles rationally equivalent to 0 , and it is denoted $A_{p}(Y)$. The Chow group of $Y$ is the group $A_{*}(Y)=\bigoplus_{p=0}^{n} A_{p}(Y)$. Given an element $\alpha \in A_{*}(Y),\{\alpha\}_{p} \in A_{p}(Y)$ will denote the $p^{\text {th }}$ homogeneous component of $\alpha$ (if $n<p$, then $\{\alpha\}_{p}=0$ ). A subscheme $X \subseteq Y$ induces a cycle class $[X] \in A_{*}(Y)$ represented by $\sum_{i=1}^{t} m_{i} X_{i}$, where $X_{1}, \ldots, X_{t}$ are the irreducible components of $X$ and $m_{1}, \ldots, m_{t}$ their geometric multiplicities in $X$. In particular, $[\emptyset]=0$. If $\alpha \in A_{*}(X)$, we will at times consider $\alpha$ to be an element of $A_{*}(Y)$, omitting in the notation the push-forward under the inclusion map.

For a rank $\rho$ vector bundle $E$ on $Y$ we have the Chern class operations $c_{i}(E)$ : $A_{p}(Y) \rightarrow A_{p-i}(Y)$ for $i \leq p, 0 \leq i \leq \rho$ and $0 \leq p \leq n$, see [5] Chapter 3. The value of $c_{i}(E)$ on $\alpha \in A_{p}(Y)$ is denoted $c_{i}(E) \cap \alpha$. The corresponding map $A_{*}(Y) \rightarrow A_{*}(Y)$ is also denoted $c_{i}(E)$, where $c_{i}(E) \cap \alpha=0$ if $\alpha \in A_{p}(Y)$ and $p<i$. The total Chern class operation $c(E): A_{*}(Y) \rightarrow A_{*}(Y): \alpha \mapsto c(E) \cap \alpha$ is defined by $c(E)=\sum_{i} c_{i}(E)$. The operation of a product of Chern classes on the Chow group is defined as the composition of the individual Chern class operations. The map $c_{0}(E)$ is the identity homomorphism. If $Y$ is smooth, the Chern classes are well defined elements of $A_{*}(Y)$ and the operations $c_{i}(E) \cap \alpha$ correspond to the intersection product. Let $X \subseteq Y$ be a closed subscheme with inclusion $i: X \rightarrow Y$ and let $\alpha \in A_{*}(X)$. By the notational convention mentioned above we will sometimes write $c(E) \cap \alpha$ to mean $c(E) \cap i_{*}(\alpha) \in A_{*}(Y)$. By the projection formula, see [5] Theorem $3.2(\mathrm{c}), c(E) \cap i_{*}(\alpha)=i_{*}\left(c\left(i^{*} E\right) \cap \alpha\right)$.

For a Cartier divisor $D$ on $Y$, the corresponding line bundle on $Y$ is denoted by $\mathcal{O}_{Y}(D)$.

In this paper, varieties are by definition irreducible and reduced. Finally, we use the convention $\operatorname{dim}(\emptyset)=-1$.

2.2. Regular embeddings. Let $Y$ be an algebraic scheme over $\mathbb{C}$. A closed embedding $X \rightarrow Y$ of a subscheme $X$ of $Y$ is called a regular embedding of codimension $d$ if the following holds. Every point of $X$ has an affine open neighborhood $U$ in $Y$ such that the ideal defining $X \cap U$ is generated by a regular sequence of length $d$ in the coordinate ring of $U$. If, for some $d, X$ is a regular embedding in $Y$ of codimension $d$, then we will simply say that the embedding is regular.

Lemma 2.1. Let $Y$ be a complex variety and let $\mathcal{L}$ be a line bundle on $Y$ such that the corresponding complete linear system is base point free. For global sections $\sigma_{1}, \ldots, \sigma_{\mu} \in \Gamma(Y, \mathcal{L})$ of $\mathcal{L}$, let $X=X\left(\sigma_{1}, \ldots, \sigma_{\mu}\right)$ denote the scheme of common zeros of $\sigma_{1}, \ldots, \sigma_{\mu}$. Then, for general $\sigma_{1}, \ldots, \sigma_{\mu} \in \Gamma(Y, \mathcal{L})$, the natural embedding $X \rightarrow Y$ is regular.

Proof. Let $\phi: Y \rightarrow \mathbb{P}^{r}$ be the map given by the complete linear system corresponding to $\mathcal{L}$ and put $e=\operatorname{dim}(\operatorname{im}(\phi))$. If $\mu>e$ then $X\left(\sigma_{1}, \ldots, \sigma_{\mu}\right)=\emptyset$ for general sections $\sigma_{1}, \ldots, \sigma_{\mu}$ and hence we may assume that $\mu \leq e$. That $X\left(\sigma_{1}\right) \rightarrow Y$ is 
a regular embedding for a general section $\sigma_{1}$ is clear since $\mathcal{O}_{Y}(U)$ is an integral domain for any open set $U \subseteq Y$. Hence, for any affine open $U \subseteq Y, \mathcal{O}_{Y}(U)$ has no zero divisors and any $\sigma_{1} \neq 0$ will give a regular sequence in $\mathcal{O}_{Y}(U)$. If $\mu=1$ we are done. If $\operatorname{dim}(Y) \leq 1$ then $e \leq 1$ and therefore $\mu=1$ in this case. Assume that $1<\mu$, in particular $1<\operatorname{dim}(Y)$. Then $e \geq 2$ and it follows from Bertini type theorems that $X\left(\sigma_{1}\right)$ is a variety for a general section $\sigma_{1}$. In fact, $X\left(\sigma_{1}\right)$ is reduced by [6] Corollary 3.4.9 and $X\left(\sigma_{1}\right)$ is irreducible by [6] 3.4.10. Replacing $Y$ by $X\left(\sigma_{1}\right)$ and restricting $\mathcal{L}$ to $X\left(\sigma_{1}\right)$ we have reduced to the case of a lower dimensional ambient variety since the composition of two regular embeddings is regular (see [5] Appendix B.7.4).

2.3. Segre classes and intersection products. Let $Y$ be a complex variety and let $X$ be an $n$-dimensional subscheme of $Y$. Suppose that $X \neq Y$ and let $\tilde{Y}$ be the blow-up of $Y$ along $X$. Let $\pi: \widetilde{Y} \rightarrow Y$ be the projection, let $\widetilde{X}=\pi^{-1}(X)$ be the exceptional divisor, and let $\eta=\left.\pi\right|_{\tilde{X}}$. The total Segre class $s(X, Y)$ of $X$ in $Y$ is an element of $A_{*}(X)$ which may be characterized as follows (see [5] Corollary 4.2.2):

$$
s(X, Y)=\sum_{p \geq 1}(-1)^{p-1} \eta_{*}\left(\widetilde{X}^{p}\right) .
$$

Here $\widetilde{X}^{p}$ is the self intersection of Cartier divisors defined in [5] Definition 2.4.2.

Remark 2.2. The Cartier divisor $\widetilde{X}$ on $\widetilde{Y}$ corresponds to a line bundle on $\widetilde{Y}$ whose restriction to $\widetilde{X}$ is the normal bundle of $\widetilde{X}$ in $\widetilde{Y}$. The dual bundle to the normal bundle is denoted $\mathcal{O}(1)$. The exceptional divisor $\tilde{X}$ is naturally identified with the so-called projective normal cone $P\left(C_{X} Y\right)$ of $X$ in $Y$. The total Segre class is given by

$$
s(X, Y)=\sum_{i \geq 0} \eta_{*}\left(c_{1}(\mathcal{O}(1))^{i} \cap\left[P\left(C_{X} Y\right)\right]\right) .
$$

This definition of Segre classes generalizes beyond normal cones of subschemes to arbitrary cones, see [5] Chapter 4.

We will now recall the Fulton-MacPherson approach to intersection products, see 5] Chapter 6 . Let $Y$ and $V$ be complex varieties and put $k=\operatorname{dim}(V)$. Let $X$ be a closed subscheme of $Y$ such that there is a regular embedding $i: X \rightarrow Y$ of codimension $d \leq k$. Let $f: V \rightarrow Y$ be a morphism and put $W=f^{-1}(X)$. Then we get the following fibre product diagram

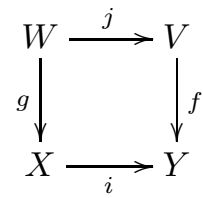

where $j: W \rightarrow V$ is the inclusion and $g: W \rightarrow X$ the restriction of $f$ to $W$. The normal cone $C_{W} V$ of $W$ in $V$ can be constructed as follows. Suppose first that $V$ is affine with coordinate ring $A$ and that $W$ is defined by an ideal $J \subseteq A$ generated by $f_{1}, \ldots, f_{d} \in A$. Let $B=A / J$. Then $C_{W} V$ is the spectrum of the $B$-algebra $\bigoplus_{s \geq 0} J^{s} / J^{s+1}$. Thus $C_{W} V$ may be embedded as a closed subscheme of $W \times \mathbb{C}^{d}$ defined by the kernel of the surjective homomorphism

$$
B\left[x_{1}, \ldots, x_{d}\right] \rightarrow \bigoplus_{s \geq 0} J^{s} / J^{s+1}
$$


which maps $x_{i}$ to the image of $f_{i}$ in $J / J^{2}$. In the general case, $C_{W} V$ may be constructed by covering $V$ with open affine subsets and gluing the normal cones of the affine patches together. In case the embedding $W \rightarrow V$ is regular, $C_{W} V$ is a vector bundle, namely the normal bundle $N_{W} V$. In this case the total Segre class of $W$ in $V$ is the inverse of the total Chern class of $N_{W} V$ in the sense that $s(W, V)=c\left(N_{W} V\right)^{-1} \cap[W]$, where $c\left(N_{W} V\right)^{-1}$ is a formal inverse of $c\left(N_{W} V\right)$. The normal cone $C_{W} V$ has pure dimension $k$, see [5] Appendix B.6.6. Let $C=C_{W} V$, let $N=g^{*}\left(N_{X} Y\right.$ ) (where $N_{X} Y$ is the normal bundle of $X$ in $Y$ ), and let $p: N \rightarrow W$ be the projection. It is shown in [5] Chapter 6, that $C$ embeds in $N$ and therefore it determines a class $[C] \in A_{k}(N)$. Now, by [5] Theorem 3.3 (a), the flat-pullback $p^{*}: A_{k-d}(W) \rightarrow A_{k}(N)$ is an isomorphism. This map is given by $p^{*}(Z)=\left[p^{-1}(Z)\right]$ for an irreducible subvariety $Z \subseteq W$. The intersection product of $V$ by $X$ on $Y$ is a class in $A_{k-d}(W)$ denoted $X \cdot V$ and defined by

$$
X \cdot V=\left(p^{*}\right)^{-1}([C]) .
$$

An important connection to Segre classes is given by [5] Proposition 6.1 (a):

$$
X \cdot V=\{c(N) \cap s(W, V)\}_{k-d} .
$$

We will now state the Residual Intersection Formula from [5] which is the main result underpinning our method to compute Segre classes. Let $Y, X, W, V, N, k$ and $d$ be as in the above definition of the intersection product $X \cdot V$. Let $Z \subseteq W$ be a closed subscheme and suppose that $Z \neq V$. Let $\pi: \widetilde{V} \rightarrow V$ be the blow-up of $V$ along $Z$ and put $\widetilde{W}=\pi^{-1}(W)$ and $\widetilde{Z}=\pi^{-1}(Z)$. Let $\widetilde{R}$ be the residual scheme to $\widetilde{Z}$ in $\widetilde{W}$ with respect to $\widetilde{V}$, see 5 Definition 9.2.1. This is a scheme such that, if $\mathscr{I}(\widetilde{Z}), \mathscr{I}(\widetilde{W})$ and $\mathscr{I}(\widetilde{R})$ are the ideal sheaves in $\mathcal{O}_{\widetilde{V}}$ defining the respective schemes, then

$$
\mathscr{I}(\widetilde{W})=\mathscr{I}(\widetilde{Z}) \cdot \mathscr{I}(\widetilde{R}) .
$$

Let $\eta: \widetilde{W} \rightarrow W$ be the restriction of $\pi$ to $\widetilde{W}$ and let $\mathcal{O}(-\widetilde{Z})$ denote the pullback of $\mathcal{O}_{\widetilde{V}}(-\widetilde{Z})$ under the inclusion $\widetilde{W} \rightarrow \widetilde{V}$.

The following proposition is Corollary 9.2.3 of [5].

Proposition 2.3. With notation as above,

$$
\begin{array}{r}
X \cdot V=\{c(N) \cap s(Z, V)\}_{k-d}+\mathbb{R}, \\
\text { where } \mathbb{R}=\eta_{*}\left(\left\{c\left(\eta^{*} N \otimes \mathcal{O}(-\widetilde{Z})\right) \cap s(\widetilde{R}, \widetilde{V})\right\}_{k-d}\right) .
\end{array}
$$

\section{Computing Segre Classes of Projective schemes}

Let $Z$ be a proper $n$-dimensional subscheme of complex projective space $\mathbb{P}^{k}$. This paper is about a method for computing the push-forward of $s\left(Z, \mathbb{P}^{k}\right)$ to $\mathbb{P}^{k}$ given an ideal defining $Z$. In this section we explain how to derive information about the push-forward given sufficiently general elements from the ideal.

Let $s_{0}, \ldots, s_{n}$ be the homogeneous components of $s\left(Z, \mathbb{P}^{k}\right)$ with $s_{i}$ of codimension $i$, that is $s\left(Z, \mathbb{P}^{k}\right)=\sum_{i=0}^{n} s_{i}$ with $s_{i} \in A_{n-i}(Z)$. The degree of a 0 -cycle $\alpha=$ $\sum_{i} m_{i} p_{i}$ on $\mathbb{P}^{k}, m_{i} \in \mathbb{Z}$ and $p_{i} \in \mathbb{P}^{k}$, is simply $\operatorname{deg}(\alpha)=\sum_{i} m_{i}$. If $\gamma: Z \rightarrow \mathbb{P}^{k}$ is the inclusion map, we define the degree of $s_{i}$ by

$$
\operatorname{deg}\left(s_{i}\right)=\operatorname{deg}\left(\gamma_{*}\left(s_{i}\right) \cdot H^{n-i}\right),
$$

where $H \in A_{k-1}\left(\mathbb{P}^{k}\right)$ is the hyperplane class and the product is the intersection product on $\mathbb{P}^{k}$. The numbers $\left\{\operatorname{deg}\left(s_{i}\right)\right\}_{i}$ is the output of our procedure, they carry 
the same information as the push-forward $\gamma_{*}\left(s\left(Z, \mathbb{P}^{k}\right)\right)$. The degree of any $\alpha \in$ $A_{p}\left(\mathbb{P}^{k}\right)$ is defined similarly by $\operatorname{deg}\left(\alpha \cdot H^{p}\right)$.

Let $I \subseteq \mathbb{C}\left[x_{0}, \ldots, x_{k}\right]$ be a homogeneous ideal. For a positive integer $m$, we use $I(m)$ to denote the $m^{t h}$ graded piece of $I$. Given a homogeneous ideal $J$, the ideal quotient $J: I$ is given by

$$
J: I=\left\{f \in \mathbb{C}\left[x_{0}, \ldots, x_{k}\right]: f I \subseteq J\right\},
$$

and the saturation of $J$ with respect to $I$ is

$$
J: I^{\infty}=\bigcup_{p \geq 1} J: I^{p} .
$$

Note that $\left(J: I^{p}\right): I=J: I^{p+1}$ for $p \geq 1$, that the ascending sequence of ideal quotients $J: I \subseteq J: I^{2} \subseteq J: I^{3} \subseteq \ldots$ stabilizes and that $J: I^{p}=J: I^{\infty}$ for large enough $p$.

Remark 3.1. Let $I$ and $J$ be homogeneous ideals of $\mathbb{C}\left[x_{0}, \ldots, x_{k}\right]$ and let $V(I)$ and $V(J)$ denote the corresponding zero-loci in $\mathbb{P}^{k}$. If $I=\mathbb{C}\left[x_{0}, \ldots, x_{k}\right]$, then $J: I^{\infty}=J$. Suppose $I \neq \mathbb{C}\left[x_{0}, \ldots, x_{k}\right]$. The ideal $J: I^{\infty}$ is homogeneous and the scheme $R$ defined by $J: I^{\infty}$ is supported on the Zariski-closure of $V(J) \backslash V(I)$. In fact, if $J=\bigcap_{i} Q_{i}$ is a primary decomposition (so each $Q_{i}$ is homogeneous and primary) then

$$
J: I^{\infty}=\bigcap_{\left\{i: V\left(Q_{i}\right) \nsubseteq V(I)\right\}} Q_{i} .
$$

To see this, note that $J: I^{\infty}=\bigcap_{i}\left(Q_{i}: I^{\infty}\right)$ and that $V\left(Q_{i}\right) \subseteq V(I)$ precisely when $\sqrt{Q_{i}} \supseteq I$. If $\sqrt{Q_{i}} \supseteq I$, then $Q_{i} \supseteq I^{p}$ for some $p$ and $Q_{i}: I^{\infty}=\mathbb{C}\left[x_{0}, \ldots, x_{k}\right]$. On the other hand, if $\sqrt{Q_{i}} \nsupseteq I$, then $Q_{i}: I=Q_{i}$ since $Q_{i}$ is primary. It follows that $Q_{i}: I^{\infty}=Q_{i}$ in this case.

The following theorem is a Bézout like equality which gives rise to a recursive formula for the degrees of the Segre classes of $Z$ in $\mathbb{P}^{k}$. Using the statement of the theorem, we may express the degree of a Segre class $s_{p}$ in terms of $\operatorname{deg}\left(s_{i}\right)$ for $i<p$ and the degree of a certain residual scheme $R$. Computing the degree of the residual $R$ is the main computational step in the method.

Theorem 3.2. Let $Z \subset \mathbb{P}^{k}$ be a subscheme of dimension $n$ defined by a non-zero homogeneous ideal $I \subseteq \mathbb{C}\left[x_{0}, \ldots, x_{k}\right]$. Let $s_{0}, \ldots, s_{n}$ denote the Segre classes of $Z$ in $\mathbb{P}^{k}$. Let $g_{0}, \ldots, g_{r}$ be a set of non-zero homogeneous generators of $I$ and put $m=\max _{i}\left\{\operatorname{deg}\left(g_{i}\right)\right\}$. For $k-n \leq d \leq k$ and general elements $f_{1}, \ldots, f_{d} \in I(m)$, the following holds. If $J$ is the ideal generated by $\left\{f_{1}, \ldots, f_{d}\right\}$ and $R \subseteq \mathbb{P}^{k}$ is the subscheme defined by $J: I^{\infty}$, then

$$
m^{d}=\operatorname{deg}(R)+\sum_{i=0}^{p}\left(\begin{array}{c}
d \\
p-i
\end{array}\right) m^{p-i} \operatorname{deg}\left(s_{i}\right),
$$

where $p=d-(k-n)$.

Proof. The proof is divided in steps 0 through 4.

Step 0: setup. Let $I^{\prime}$ be the ideal generated by $I(m)$ and let $\mathfrak{m}=\left(x_{0}, \ldots, x_{k}\right)$. Then $I: \mathfrak{m}^{\infty}=I^{\prime}: \mathfrak{m}^{\infty}$, and therefore $I$ and $I^{\prime}$ define the same scheme $Z \subseteq \mathbb{P}^{k}$. We may thus assume that $g_{0}, \ldots, g_{r}$ all have degree $m$. Let

$$
\pi: \widetilde{\mathbb{P}}^{k} \rightarrow \mathbb{P}^{k}
$$


be the blow-up of $\mathbb{P}^{k}$ along $Z$ and put $\widetilde{Z}=\pi^{-1}(Z)$. The map $\mathbb{P}^{k} \backslash Z \rightarrow \mathbb{P}^{r}$ defined by $g_{0}, \ldots, g_{r}$ extends to a map

$$
\phi: \widetilde{\mathbb{P}}^{k} \rightarrow \mathbb{P}^{r},
$$

see [5] 4.4. In fact, $\widetilde{\mathbb{P}}^{k}$ embeds in $\mathbb{P}^{k} \times \mathbb{P}^{r}$ in such a way that $\left(\widetilde{\mathbb{P}}^{k} \backslash \widetilde{Z}\right)$ is the graph of the map $\mathbb{P}^{k} \backslash Z \rightarrow \mathbb{P}^{r}$ and $\phi$ is the projection. Let $W \subseteq \mathbb{P}^{k}$ be the scheme defined by $f_{1}, \ldots, f_{d}$ and put $\widetilde{W}=\pi^{-1}(W)$. Let $\widetilde{R}$ be the residual to $\widetilde{Z}$ in $\widetilde{W}$ with respect to $\widetilde{\mathbb{P}}^{k}$.

Step 1: we will show that $\widetilde{R} \rightarrow \widetilde{\mathbb{P}}^{k}$ is a regular embedding and that $\widetilde{R}$ is either empty or of pure dimension $k-d$ and that no irreducible component of $\widetilde{R}$ is contained in $\widetilde{Z}$. By [5] 4.4.,

$$
\phi^{*}\left(\mathcal{O}_{\mathbb{P}^{r}}(1)\right)=\pi^{*}\left(\mathcal{O}_{\mathbb{P}^{k}}(m)\right) \otimes \mathcal{O}_{\widetilde{\mathbb{P}} k}(-\widetilde{Z}) .
$$

In concrete terms, $\widetilde{\mathbb{P}}^{k}$ is defined by a bi-homogeneous ideal

$$
K \subseteq \mathbb{C}\left[x_{0}, \ldots, x_{k}\right]\left[y_{0}, \ldots, y_{r}\right]
$$

such that $K$ contains the elements $g_{i} y_{j}-g_{j} y_{i}$ for $0 \leq i<j \leq r$. Observe that $\widetilde{Z}$ is given by the vanishing of $g_{0}, \ldots, g_{r}$. Consider the affine open set

$$
U=U_{\alpha \beta}=\left\{\left(x_{0}, \ldots, x_{k}, y_{0}, \ldots, y_{r}\right) \in \widetilde{\mathbb{P}}^{k}: x_{\alpha} \neq 0, y_{\beta} \neq 0\right\}
$$

and let $w_{0}=\frac{y_{0}}{y_{\beta}}, \ldots, w_{r}=\frac{y_{r}}{y_{\beta}}$ with $w_{\beta}=1$. Then $w_{0}, \ldots, \widehat{w_{\beta}}, \ldots, w_{r}$ are coordinates on $\mathbb{C}^{r}=\left\{y_{\beta} \neq 0\right\} \subset \mathbb{P}^{r}$. Note that for all $i, f_{i}=\sum_{j=0}^{r} \lambda_{i}^{j} g_{j}$, for a general vector $\left(\lambda_{i}^{0}, \ldots, \lambda_{i}^{r}\right) \in \mathbb{C}^{r+1}$. With an abuse of notation, we use $g_{i}, f_{i}$ and $w_{i}$ to denote the corresponding elements of the coordinate ring of $U$. Then, $g_{j}=w_{j} g_{\beta}$ for all $j$. Hence $\widetilde{Z} \cap U$ is defined by $g_{\beta}$. Also, $f_{i}=\left(\sum_{j=0}^{r} \lambda_{i}^{j} w_{j}\right) g_{\beta}$. It follows that $\widetilde{R} \cap U$ is defined by the ideal $\left(\sum_{j=0}^{r} \lambda_{1}^{j} w_{j}, \ldots, \sum_{j=0}^{r} \lambda_{d}^{j} w_{j}\right)$. We conclude that $\widetilde{R}=\phi^{-1}(L)$ for a general linear subspace $L \subseteq \mathbb{P}^{r}$ of codimension $d$ (if $r<d$, then $\widetilde{R}=\emptyset$ ). Hence $\widetilde{R}$ is either empty or of pure dimension $k-d$ and $\widetilde{R} \cap \widetilde{Z}$ is either empty or of pure dimension $k-d-1$. It follows that no irreducible component of $\widetilde{R}$ is contained in $\widetilde{Z}$. Since $\widetilde{\mathbb{P}}^{k}$ is a variety (see [8] Proposition II.7.16 or [5] Appendix B.6.4), it follows by Lemma 2.1 that the embedding of $\widetilde{R}$ in $\widetilde{\mathbb{P}}^{k}$ is regular.

Step 2: applying Proposition 2.3. Let $X_{1}, \ldots, X_{d}$ be defined by $X_{\nu}=\left\{f_{\nu}=0\right\}$. Then $W=\bigcap_{\nu=1}^{d} X_{\nu}$. Let $X=X_{1} \times \cdots \times X_{d}$ and $Y=\mathbb{P}^{k} \times \cdots \times \mathbb{P}^{k}$ ( $d$ factors). Let $j: W \rightarrow \mathbb{P}^{k}$ be the inclusion and let $f: \mathbb{P}^{k} \rightarrow Y$ and $g: W \rightarrow X$ be the diagonal morphisms. The morphism $i: X \rightarrow Y$ induced by the inclusions $X_{1}, \ldots, X_{d} \subset \mathbb{P}^{k}$ is a regular embedding of codimension $d$. Put $N=g^{*}\left(N_{X} Y\right)$. Letting $V=\mathbb{P}^{k}$, we apply Proposition 2.3 to the diagram



and conclude that

$$
X \cdot \mathbb{P}^{k}=\left\{c(N) \cap s\left(Z, \mathbb{P}^{k}\right)\right\}_{k-d}+\mathbb{R} .
$$

Here $s\left(Z, \mathbb{P}^{k}\right)$ is regarded as a rational equivalence class on $W$. 
Step 3: we will show that

$$
m^{d}=\sum_{i=0}^{p}\left(\begin{array}{c}
d \\
p-i
\end{array}\right) m^{p-i} \operatorname{deg}\left(s_{i}\right)+\operatorname{deg}\left(j_{*}(\mathbb{R})\right),
$$

where $p=d-(k-n)$. We shall first see that $N=j^{*}(E)$ where $E=\bigoplus_{\nu=1}^{d} \mathcal{O}_{\mathbb{P}^{k}}(m)$. Let $p_{\nu}: Y \rightarrow \mathbb{P}^{k}$ be the $\nu^{\text {th }}$ projection and let $\mathbb{X}_{\nu}$ be the divisor $p_{\nu}^{-1}\left(X_{\nu}\right)$ on $Y$. Then, by [5] B.7.4,

$$
N_{X} Y=\left.\bigoplus_{\nu=1}^{d} \mathcal{O}_{Y}\left(\mathbb{X}_{\nu}\right)\right|_{X}
$$

Since $f^{*}\left(\mathcal{O}_{Y}\left(\mathbb{X}_{\nu}\right)\right)=\mathcal{O}_{\mathbb{P}^{k}}(m)$ for all $\nu$, we have that

$$
j^{*}\left(\mathcal{O}_{\mathbb{P}^{k}}(m)\right)=(f \circ j)^{*}\left(\mathcal{O}_{Y}\left(\mathbb{X}_{\nu}\right)\right)=(i \circ g)^{*}\left(\mathcal{O}_{Y}\left(\mathbb{X}_{\nu}\right)\right)=g^{*}\left(\left.\mathcal{O}_{Y}\left(\mathbb{X}_{\nu}\right)\right|_{X}\right)
$$

for all $\nu$. Hence

$$
N=g^{*}\left(N_{X} Y\right)=\bigoplus_{\nu=1}^{d} g^{*}\left(\left.\mathcal{O}_{Y}\left(\mathbb{X}_{\nu}\right)\right|_{X}\right)=j^{*} E .
$$

Note that $c(E)=(1+m H)^{d} \in A_{*}\left(\mathbb{P}^{k}\right)$, where $H \in A_{k-1}\left(\mathbb{P}^{k}\right)$ is the hyperplane class.

We now push both sides of (2) forward to $\mathbb{P}^{k}$ by $j$ and then take degrees. By Bézout's theorem, $\operatorname{deg}\left(j_{*}\left(X \cdot \mathbb{P}^{k}\right)\right)=m^{d}$ (see [5] Example 6.2.6). By the projection formula, $j_{*}\left(c(N) \cap s\left(Z, \mathbb{P}^{k}\right)\right)=c(E) \cap j_{*}\left(s\left(Z, \mathbb{P}^{k}\right)\right)$. We get that

$$
\begin{gathered}
j_{*}\left(\left\{c(N) \cap s\left(Z, \mathbb{P}^{k}\right)\right\}_{k-d}\right)=\left\{j_{*}\left(c(N) \cap s\left(Z, \mathbb{P}^{k}\right)\right)\right\}_{k-d}= \\
\left\{(1+m H)^{d} \cdot j_{*}\left(s\left(Z, \mathbb{P}^{k}\right)\right)\right\}_{k-d}=\left\{(1+m H)^{d} \cdot \sum_{i=0}^{n} j_{*}\left(s_{i}\right)\right\}_{k-d} .
\end{gathered}
$$

The degree of the latter expression is

$$
\sum_{i=0}^{p}\left(\begin{array}{c}
d \\
p-i
\end{array}\right) m^{p-i} \operatorname{deg}\left(s_{i}\right)
$$

where $p=d-(k-n)$.

Step 4: it remains to see that $\operatorname{deg}\left(j_{*}(\mathbb{R})\right)=\operatorname{deg}(R)$ where $R \subseteq \mathbb{P}^{k}$ is the scheme defined by $J: I^{\infty}$. In fact, we shall see that $j_{*}(\mathbb{R})=[R]$ in $A_{*}\left(\mathbb{P}^{k}\right)$. Let $\eta: \widetilde{W} \rightarrow W$ be the restriction of $\pi$ to $\widetilde{W}$. Since $\widetilde{R} \rightarrow \widetilde{\mathbb{P}}^{k}$ is a regular embedding we have that $s\left(\widetilde{R}, \widetilde{\mathbb{P}}^{k}\right)=c\left(N_{\widetilde{R}} \widetilde{\mathbb{P}}^{k}\right)^{-1} \cap[\widetilde{R}]$. Since $\widetilde{R}$ is either empty or has pure dimension $k-d$,

$$
\begin{gathered}
\left\{c\left(\eta^{*} N \otimes \mathcal{O}(-\widetilde{Z})\right) \cap s\left(\widetilde{R}, \widetilde{\mathbb{P}}^{k}\right)\right\}_{k-d}= \\
\left\{c\left(\eta^{*} N \otimes \mathcal{O}(-\widetilde{Z})\right) \cap\left(c\left(N_{\widetilde{R}} \widetilde{\mathbb{P}}^{k}\right)^{-1} \cap[\widetilde{R}]\right)\right\}_{k-d}=[\widetilde{R}] .
\end{gathered}
$$

Hence $\mathbb{R}=\eta_{*}([\widetilde{R}])$. Let $\widetilde{R}_{1}, \ldots, \widetilde{R}_{t}$ be the irreducible components of $\widetilde{R}$ and let $m_{1}, \ldots, m_{t}$ denote their geometric multiplicities. Since none of the components $\widetilde{R}_{1}, \ldots, \widetilde{R}_{t}$ is contained in $\widetilde{Z}$ and $\pi: \widetilde{\mathbb{P}}^{k} \rightarrow \mathbb{P}^{k}$ is an isomorphism outside $\widetilde{Z}, j_{*}(\mathbb{R})=$ $\sum_{i=1}^{t} m_{i}\left[\pi\left(\widetilde{R}_{i}\right)\right]$. Observe that $\pi$ induces an isomorphism $(\widetilde{R} \backslash \widetilde{Z}) \cong(W \backslash Z)$. It follows from (1) that $\sum_{i=1}^{t} m_{i}\left[\pi\left(\widetilde{R}_{i}\right)\right]=[R]$. 
Remark 3.3. With notation as in the proof of Theorem 3.2 note that the group $\mathrm{GL}\left(\mathbb{C}^{k+1}\right) \times \mathrm{GL}\left(\mathbb{C}^{r+1}\right)$ acts transitively on $\mathbb{P}^{k} \times \mathbb{P}^{r}$ and that $\widetilde{\mathbb{P}}^{k}$ is regular outside $\widetilde{Z}$. It follows by Kleiman's transversality theorem 10 that for a general linear subspace $L \subseteq \mathbb{P}^{r}$ of codimension $d, \widetilde{R}=\widetilde{\mathbb{P}}^{k} \cap\left(\mathbb{P}^{k} \times L\right)$ is regular outside $\widetilde{Z}$ and the multiplicities $m_{1}, \ldots, m_{t}$ of the components of $\widetilde{R}$ are all equal to 1 . Moreover, it follows that the scheme $R$ defined by $J: I^{\infty}$ is regular outside $Z$. This could be of interest in connection with the computation of the degree of $R$, which is the main computational ingredient in our method to compute Segre classes.

\section{THE METHOD}

Theorem 3.2 states that certain conditions hold for a general choice of elements of a given ideal. By choosing these elements randomly we turn this into a probabilistic algorithm. Applying Theorem 3.2 to solve for the Segre classes recursively, we obtain the following procedure to compute the degrees of the Segre classes of a subscheme of projective space. The input is an ideal defining the subscheme.

Procedure $1 \mathrm{~A}$ method to compute the degrees of Segre classes

Input: Non-zero homogeneous generators $g_{0}, \ldots, g_{r}$ of an ideal $I \subseteq \mathbb{C}\left[x_{0}, \ldots, x_{k}\right]$.

Output: The degrees of the Segre classes of the subscheme of $\mathbb{P}^{k}$ defined by $I$.

1: Let $m=\max _{i}\left\{\operatorname{deg}\left(g_{i}\right)\right\}$.

2: Let $Z \subset \mathbb{P}^{k}$ be the scheme defined by $I$ and compute $n=\operatorname{dim}(Z)$.

3: Pick random elements $f_{1}, \ldots, f_{k} \in I(m)$.

4: for $d=k-n$ to $k$ do

Let $J=\left(f_{1}, \ldots, f_{d}\right)$.

Compute $\operatorname{deg}(R)$, where $R \subseteq \mathbb{P}^{k}$ is the scheme defined by $J: I^{\infty}$.

7: Let $p=d-(k-n)$ and compute

$$
\operatorname{deg}\left(s_{p}\right)=m^{d}-\operatorname{deg}(R)-\sum_{i=0}^{p-1}\left(\begin{array}{c}
d \\
p-i
\end{array}\right) m^{p-i} \operatorname{deg}\left(s_{i}\right) .
$$

: end for

9: return $\operatorname{deg}\left(s_{0}\right), \ldots, \operatorname{deg}\left(s_{n}\right)$

Remark 4.1. We will use the notation of Procedure 1 and the proof of Theorem 3.2 In particular $\pi: \widetilde{\mathbb{P}}^{k} \rightarrow \mathbb{P}^{k}$ denotes the blow-up of $\mathbb{P}^{k}$ along $Z$. Instead of saturating with respect to the whole ideal $I$, as is done in Procedure 1, one could saturate with respect to one element of $I$. Let $h \in I, h \neq 0$, let $R^{\prime}$ be the scheme defined by $J:(h)^{\infty}$ and let $H \subseteq \mathbb{P}^{k}$ be the hypersurface defined by $h$. The claim is that we could replace $R$ by $R^{\prime}$ in Procedure 1. Tracing back the conditions on $R$ used in the proof of Theorem 3.2 we see that we only need to show that no irreducible component of the residual $\widetilde{R} \subseteq \widetilde{\mathbb{P}}^{k}$ is contained in $\pi^{-1}(H)$. This follows exactly as in step 1 of the proof of Theorem 3.2 where it is shown that $\widetilde{R}$ has no irreducible component inside the exceptional divisor $\widetilde{Z}$.

Remark 4.2. Let $Z \subseteq \mathbb{P}^{k}$ be a subscheme of dimension $n$ and let $s_{i} \in A_{n-i}(Z)$ be the Segre classes of $Z$, that is $s\left(Z, \mathbb{P}^{k}\right)=\sum_{i=0}^{n} s_{i}$. Define the total Chern-Fulton class of $Z$ by

$$
c^{\prime}(Z)=c\left(\left.T_{\mathbb{P}^{k}}\right|_{Z}\right) \cap s\left(Z, \mathbb{P}^{k}\right),
$$


where $T_{\mathbb{P}^{k}}$ is the tangent bundle of $\mathbb{P}^{k}$. This definition is independent of the embedding of $Z$ in $\mathbb{P}^{k}$ in the sense that if $Z$ admits two embeddings into smooth varieties $M$ and $P$, then $c\left(\left.T_{M}\right|_{Z}\right) \cap s(Z, M)=c\left(\left.T_{P}\right|_{Z}\right) \cap s(Z, P)$, see [5] Example 4.2.6. Let $c^{\prime}(Z)=\sum_{i=0}^{n} c_{i}^{\prime}$, with $c_{i}^{\prime} \in A_{n-i}(Z)$. If $Z$ is smooth, then the Chern-Fulton classes coincide with the Chern classes of the tangent bundle. Since $c\left(T_{\mathbb{P}^{k}}\right)=(1+H)^{k+1}$ where $H \in A_{k-1}\left(\mathbb{P}^{k}\right)$ is the hyperplane class, the degrees of the Segre classes and those of the Chern-Fulton classes are related by

$$
\operatorname{deg}\left(c_{i}^{\prime}\right)=\sum_{p=0}^{i}\left(\begin{array}{c}
k+1 \\
i-p
\end{array}\right) \operatorname{deg}\left(s_{p}\right) .
$$

Recall that in the smooth case, the degree of the top Chern class of the tangent bundle is equal to the topological Euler characteristic. Thus, in case $Z$ is smooth, $\operatorname{deg}\left(c_{n}^{\prime}\right)$ is the topological Euler characteristic of $Z$ and Procedure 1 provides a way of computing this topological invariant.

\section{EXAMPLES}

In this section we illustrate Procedure 1 with some examples. In these examples, for an $n$-dimensional subscheme $Z \subseteq \mathbb{P}^{k}$, we use the notation $\sigma(Z)=$ $\left(\operatorname{deg}\left(s_{0}\right), \ldots, \operatorname{deg}\left(s_{n}\right)\right)$, where $s\left(Z, \mathbb{P}^{k}\right)=\sum_{i=0}^{n} s_{i}$ and $s_{i} \in A_{n-i}(Z)$.

Example 5.1. Let $I \subset \mathbb{C}[x, y, z]$ be the ideal $I=\left(x^{2}, y^{2}, x y\right)$ and let $p \in \mathbb{P}^{2}$ be the degree three zero-scheme defined by $I$. Then $s\left(p, \mathbb{P}^{2}\right)=s_{0} \in A_{0}(p)$, and $A_{0}(p) \cong \mathbb{Z}$ via the degree map. Now let $f_{1}, f_{2} \in I$ be general elements of degree 2 and put $J=\left(f_{1}, f_{2}\right)$. Then $J: I=(x, y)$ and $(J: I): I=(1)$. Hence $J: I^{\infty}=(1)$ and the residual $R$ is empty. Therefore

$$
s_{0}=2^{2}-\operatorname{deg}(R)=4 .
$$

Note that $s_{0}$ is not equal to the degree of $p$ (which is equal to 3 ).

Example 5.2. Consider a plane curve $C \subseteq \mathbb{P}^{2}$ defined by one element $g \in \mathbb{C}[x, y, z]$ of degree $m$. Then it is immediate from Procedure 1 that $\sigma(C)=\left(m,-m^{2}\right)$. In case $g=x y$, we get $\sigma(C)=(2,-4)$.

Now consider the scheme $D \subseteq \mathbb{P}^{2}$ defined by $I=\left(x^{2} y, x y^{2}\right)$. The support of $D$ is the union of the lines $L_{1}=\{x=0\}$ and $L_{2}=\{y=0\}$, but $D$ has an embedded point at $p=L_{1} \cap L_{2}$. The normal cone $C_{D} \mathbb{P}^{2}$ has three irreducible components, all of dimension 2, and the supports of these components are $L_{1}, L_{2}$ and $p$, respectively. The supports are the so-called distinguished varieties of the intersection defined by $\left\{x^{2} y, x y^{2}\right\}$. A general element $f_{1} \in I(3)$ may be written $f_{1}=x y(a x+b y)$, for general $a, b \in \mathbb{C}$. Then $\left(f_{1}\right): I^{\infty}=\left(f_{1}\right): I=(a x+b y)$. Hence the residual $R$ is the line $\{a x+b y=0\}$ and

$$
\operatorname{deg}\left(s_{0}\right)=3-\operatorname{deg}(R)=2 .
$$

For a general $f_{2} \in I(3)$ we have that $\left(f_{1}, f_{2}\right)=I$ and it follows that

$$
\operatorname{deg}\left(s_{1}\right)=3^{2}-2 \cdot 3 \operatorname{deg}\left(s_{0}\right)=-3 .
$$

In summary, $\sigma(D)=(2,-3)$. Observe that the Segre classes detect the embedded point $p$. 
Example 5.3. To illustrate Procedure 1 we show in this example how it works on a surface $Z \subseteq \mathbb{P}^{k}$. Let $Z$ be defined by an ideal $I$ which is generated by polynomials $g_{0}, \ldots, g_{r}$ in $\mathbb{C}\left[x_{0}, \ldots, x_{k}\right]$. Let $m=\max _{i}\left\{\operatorname{deg}\left(g_{i}\right)\right\}$ and let $f_{1}, \ldots, f_{k} \in I(m)$ be general. Let $J_{2}=\left(f_{1}, \ldots, f_{k-2}\right), J_{1}=\left(f_{1}, \ldots, f_{k-1}\right)$ and $J_{0}=\left(f_{1}, \ldots, f_{k}\right)$. Let $W_{i}$ be the scheme defined by $J_{i}$ and let $R_{i}$ be the scheme defined by $J_{i}: I^{\infty}$. Then

$$
\begin{array}{ll}
W_{2}=Z \cup R_{2} & \operatorname{dim}\left(R_{2}\right)=2 \text { (or } R_{2} \text { is empty), } \\
W_{1}=Z \cup R_{1} & \operatorname{dim}\left(R_{1}\right)=1 \text { (or } R_{1} \text { is empty), } \\
W_{0}=Z \cup R_{0} & \operatorname{dim}\left(R_{0}\right)=0 \text { (or } R_{0} \text { is empty). }
\end{array}
$$

The degrees of the Segre classes $s_{0}, s_{1}, s_{2}$ of $Z$ in $\mathbb{P}^{k}$ are computed as follows:

$$
\begin{aligned}
& \operatorname{deg}\left(s_{0}\right)=m^{k-2}-\operatorname{deg}\left(R_{2}\right), \\
& \operatorname{deg}\left(s_{1}\right)=m^{k-1}-\operatorname{deg}\left(R_{1}\right)-(k-1) m \operatorname{deg}\left(s_{0}\right), \\
& \operatorname{deg}\left(s_{2}\right)=m^{k}-\operatorname{deg}\left(R_{0}\right)-\left(\begin{array}{c}
k \\
2
\end{array}\right) m^{2} \operatorname{deg}\left(s_{0}\right)-k m \operatorname{deg}\left(s_{1}\right) .
\end{aligned}
$$

\section{IMPLEMENTATION AND BENCHMARKS}

There is an implementation of Procedure 1 in the symbolic setting using the software system Macaulay2 77. It uses the improvement of Remark 4.1, The user may choose to be given the degrees of the Chern-Fulton classes as output. As an alternative to the Gröbner basis computations carried out in Macaulay2, one can use the regenerative cascade algorithm [9] implemented in the software package Bertini [3]. The regenerative cascade algorithm uses numerical homotopy methods to collect data about solution sets of polynomial equations and this data includes the degrees of the residuals that are used in Procedure 1 to compute the degrees of the Segre classes. Both implementations are available at http://www.math.su.se/ jost/segreimplementation.htm.

Table 1 shows run times on some examples, comparing our implementation "segreClass" to two other algorithms. One is the April 2009 version of "CSM" which implements Aluffi's algorithm to compute Segre classes, see [1]. The other is the routine "euler" from Macaulay2 which computes the topological Euler characteristic of a smooth projective variety. Observe that the input to "euler" is a projective variety, not an ideal. Following Table 1, we provide a few details about each example. Additional details on how to generate the equations for each example may be found at http://www.math.su.se/ jost/segreimplementation.htm.

The defining equations of the rational normal curves in Table 1 are given as $(2 \times 2)$-minors of a matrix with variables as entries. The Grassmann manifold is embedded with the Plücker embedding. The surface in $\mathbb{P}^{8}$ is defined by the $(2 \times 2)$ minors of $(4 \times 3)$-matrix of random linear forms. The ideal of the Abelian surface is generated in degrees 5 and 6 . The Grassmannian and the Segre product were run over $\mathbb{Q}$ and the other examples were run over the finite field with 32749 elements.

Remark 6.1. In connection with the comparison made in Table 1 it should be noted that the routine "euler" computes the topological Euler characteristic by first computing the Hodge numbers of the variety and then taking an alternating sum of them. Thus, "euler" computes interesting information that is not attainable from the Segre classes in any obvious way. 
TABle 1. Comparison of run times. On an AMD Athlon 64 Processor, $2.2 \mathrm{GHz}$, and with $1 \mathrm{~GB}$ RAM. The computations marked with "-" were terminated after 3 hours.

\begin{tabular}{llll}
\hline Input & segreClass & CSM & euler \\
\hline Rational normal curve in $\mathbb{P}^{6}$ & $0.5 \mathrm{~s}$ & $180 \mathrm{~s}$ & $4 \mathrm{~s}$ \\
Rational normal curve in $\mathbb{P}^{10}$ & - & - & $512 \mathrm{~s}$ \\
Grassmannian $\mathbb{G}(1,5) \subseteq \mathbb{P}^{14}$ & - & $2 \mathrm{~s}$ & - \\
Smooth surface in $\mathbb{P}^{8}$ defined by minors & $89 \mathrm{~s}$ & - & - \\
Abelian surface in $\mathbb{P}^{4}$ & $175 \mathrm{~s}$ & - & - \\
Segre embedding of $\mathbb{P}^{2} \times \mathbb{P}^{3}$ in $\mathbb{P}^{11}$ & - & $8 \mathrm{~s}$ & - \\
\hline
\end{tabular}

\section{Conclusions}

This paper presents an elementary algorithm, based on residual intersection, to compute the degrees of Segre classes of a subscheme of projective space. The symbolic version of the algorithm has been implemented in Macaulay2 [7. The numerical version, using numerical homotopy methods and the regenerative cascade algorithm [9], has been implemented in the software package Bertini [3]. The table of example run times illustrate the complementary nature of the symbolic implementation of the algorithm to previous symbolic algorithms, in particular to the algorithm of Aluffi [1] in the general case and to the algorithm "euler" found in Macaulay2 when run on smooth projective varieties. The numeric implementation shows promise for extending the range of problems to which the algorithm can be applied.

\section{REFERENCES}

[1] P. Aluffi, Computing characteristic classes of projective schemes, Journal of Symbolic Computation 35 (2003), 3-19.

[2] D.J. Bates, D. Eklund, C. Peterson, Computing intersection numbers of Chern classes (submitted).

[3] D.J. Bates, J.D. Hauenstein, A.J. Sommese, C.W. Wampler, Bertini: Software for Numerical Algebraic Geometry, available at http://www.nd.edu/ sommese/bertini

[4] S. Di Rocco, D. Eklund, C. Peterson, A.J. Sommese, Chern numbers of smooth varieties via homotopy continuation and intersection theory, Journal of Symbolic Computation, Volume 46, Issue 1 (2011), 23-33.

[5] W. Fulton, Intersection Theory, Second edition. Ergebnisse der Mathematik und ihrer Grenzgebiete, 3. Folge, no. 2. Springer-Verlag, Berlin (1998).

[6] H. Flenner, L. O'Carroll, W. Vogel, Joins and Intersections, Springer Monographs in Mathematics, Springer-Verlag, Berlin (1999)

[7] D. Grayson, M. Stillman, Macaulay2: a software system for research in algebraic geometry, available at http://www.math.uiuc.edu/Macaulay2

[8] R. Hartshorne, Algebraic Geometry, Graduate Texts in Mathematics 52, Springer-Verlag, New York-Heidelberg, (1977).

[9] J.D. Hauenstein, A.J. Sommese, C.W. Wampler, Regenerative cascade homotopies for solving polynomial systems, Applied Mathematics and Computation 218 (2011), 1240-1246.

[10] S.L. Kleiman, The transversality of a general translate, Compositio Math. 28 (1974), 287297.

[11] A.J. Sommese, C.W. Wampler, The Numerical solution of systems of polynomials arising in engineering and science, World Scientific Publishing Co. Pte. Ltd., Hackensack, NJ, (2005). 
Institut Mittag-Leffler, Auravägen 17, SE-182 60 Duursholm Stockholm, Sweden

E-mail address: daek@math.kth.se

$U R L:$ http://www.math.kth.se/ daek

Department of Mathematics, Stockholm University, SE-106 91 Stockholm, Sweden

E-mail address: jost@math.su.se

$U R L:$ http://www.math.su.se/ jost/

Department of Mathematics, Colorado State University, Fort Collins, CO 80523

E-mail address: peterson@math.colostate.edu

$U R L$ : http://www. math.colostate.edu/ peterson 